\title{
TOWARDS EARTH SYSTEM PREDICTIONS: THE IMPORTANCE OF OCEAN OBSERVATIONS
}

\author{
Guy Brasseur \\ Climate Service Center at GKSS, Hamburg, Germany; and National Center for Atmospheric Research, \\ Boulder, CO,USA, Email: guy.brasseur@gkss.de
}

\begin{abstract}
Society requires specific information on climate changes and its consequences at the global and regional scales. In the past, considerable progress has been made towards the realistic simulation of important Earth system processes. Model projections for the next century have been used as an important input to the assessments conducted by the Inter-governmental Panel for Climate Change (IPCC). There is now a growing demand for shorter-term climate predictions. This represents a major challenge for the scientific community, and specifically for the newly created Climate Services that provide authoritative information on climate-related questions. Decadal predictions models require initial conditions based on observations, specifically on ocean data. It is therefore crucial that the ocean community improves its ocean observing and monitoring systems, which will soon become a key component of operational climate prediction systems. In addition to physical quantities, biogeochemical variables should also be routinely measured.
\end{abstract}

\section{INTRODUCTION}

Our planet is facing an unprecedented crisis due in large part to rapid population growth and economic development. Humans are using increasingly large amounts of natural resources including environmental goods and services (i.e., food, water, etc.). As a result, biogeochemical cycles, including the carbon and nitrogen cycles have been deeply perturbed at the local, regional and global scales. Water and air are getting increasingly polluted, while climate is changing and will continue to do so in the future. Among the key problems associated with climate change and environmental degradation are energy availability (including the search for alternative and more sustainable energy sources), water scarcity and food production, air and water quality, urbanization and population migration, human health, poverty and education. Mitigating anthropogenic climate forcing, adapting to unavoidable environmental changes, reducing the impacts of rare but extreme weather events and other ecological disturbances, managing our global environment including the ocean and its living species, are major challenges that society is facing. Science and education should provide the knowledge needed by humanity to face such key problems. In particular, there is a clear need to understand interactions and feedbacks in the entire Earth System including the relations between nature and society. Here, one should recognize the immense role played in particular by the ocean and the necessity to further investigate the dynamical, physical, chemical and biological processes that govern its evolution at different timescales. Preserving the ocean and managing adequately its resources requires intensive research. Continuous observations of key oceanic variables at the global scale and their integration in a modeling framework through data assimilation procedures are key for substantial progress.

\section{RECENT PROGRESS IN CLIMATE RESEARCH AND CHALLENGING QUESTIONS}

Over the last decades, much progress has been made in several areas of climate research. Clearly, there is a better understanding of the drivers (i.e. cause and effect) of climate change, of the complex scale interactions that affect the climate system and of the systemic interactions and feedbacks that determine its evolution. Improved global datasets (climate, atmosphere, land and oceans) and historic coverage of observational data are available. First attempts have been made to integrate natural and human processes: a wealth of global change scenarios has been developed.

Fundamental questions remain, however, to be addressed. Atmospheric components of global climate models do not yet represent explicitly individual clouds, nor do they simulate convective exchanges, specifically in the tropics. Ocean components need to be applied at higher spatial resolution so that important processes such as multi-scale eddies be explicitly represented. More work is also needed to quantify the role of ocean biogeochemistry and ecosystems in regulating climate. Specifically, it will be important to design the appropriate observational system needed to identify the key marine biogeochemical cycles and related ecosystem processes that will be impacted by global change, and to assess the responses of key biogeochemical cycles to climate change.

\section{NEW DIRECTIONS}

Climate science has made major advances during the last two decades, yet climate information is not routinely used in economic planning processes. Before 
the completion of the last IPCC assessment, the major task of the scientific community was to determine if

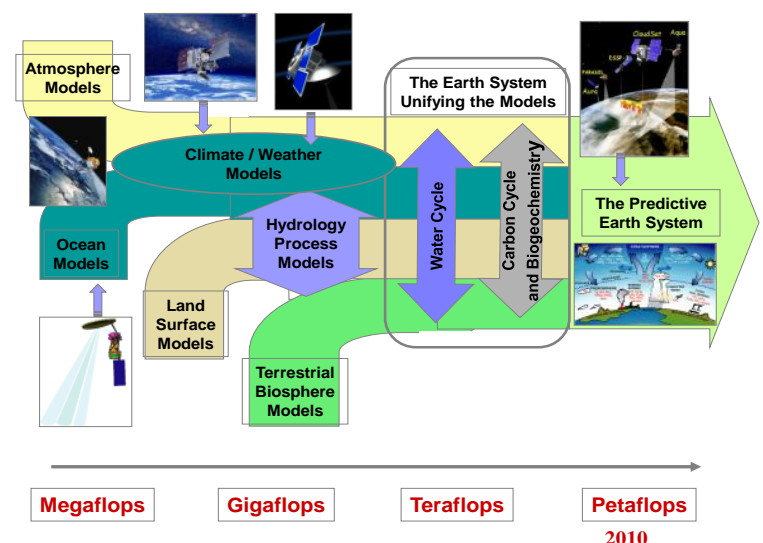

Figure 1. Schematic representation of an Earth System Model that unifies information from atmospheric, oceanic land surface and biospheric components. Hydrological and biogeochemical cycles are key elements of such an integrated model. Assimilation of observations is an important element of prediction systems. Powerful supercomputers are needed to perform simulations at the highest possible spatial resolution. The speed of supercomputers has gradually increased over the last decades.

anthropogenic climate change was occurring and if further change was expected in the decades and perhaps the centuries ahead. Coupled ocean-atmosphere general circulation models have been used to provide climate projections for different possible emission scenarios of carbon dioxide and other greenhouse gases. These models include a representation of the carbon cycle and in some cases, of the nitrogen cycles. Most of them also account for the direct and indirect effects of aerosol particles (sulfate, nitrate, black and organic carbon, dust, sea-salt, etc.) on the solar and atmospheric radiation fields and on the hydrological cycle.

As the impact of humans on climate is becoming better documented, the questions posed to the scientific community have changed. Today, they focus increasingly on climate impacts (i.e., water resources and food availability, environmental goods and services including fish production, frequency of extreme weather events, air quality and human health). A major issue is to develop response strategies, and specifically to provide to decision-makers science-related information that will lead to the most appropriate adaptation measures under different possible mitigation scenarios.

\section{CLIMATE SERVICES}

The third World Climate Conference (WCC-3), which met in Geneva in September 2009, decided to establish a Global Framework for Climate Services. The objective is to strengthen production, availability, delivery of science-based information and climate prediction. Climate Service Centers will provide reliable, well documented, authoritative and easily used information on climate issues. The rationale is to develop the most effective approaches to mitigation and adaptation strategies. Several countries have already established Climate Services. Others are discussing the best strategy to respond to a growing number of questions posed by different sectors of society.

To be successful, Climate Services will have to develop sustained, nationally and regionally-based interactions with users in different economic sectors, provide balanced, credible, cutting edge scientific and technical information, and engage a diversity of users in meaningful ways to ensure that their needs are being met. Implementation of such broad objectives will require that observations, standards and data stewardship be strengthened, and that regional and local projections of climate change be improved. Since Climate Services will build bridges between science and society, and will inform policy options, it will have to be strongly linked to research conducted in key scientific institutions.

A climate information system must be based on the several elements (Trenberth, personal communication):

1. An observational component focusing on the different climate forcings (e.g., greenhouse gases and aerosols) and on physical, chemical, and biological processes in the atmosphere, ocean, and land; a comprehensive observation system should also include socio-economic, demographic, political and cultural information.

2. An analysis component that is comprehensive and integrated; it should lead to key products needed by users.

3. An assimilation component that is based on the most advanced modeling approaches and provides the information needed to initialize climate prediction systems.

4. An attribution component that attempts to determine the cause of environmental changes

5. An assessment component designed to address global as well as regional questions, focuses on impacts of climate change and is used for planning purposes.

6. A prediction component that considers multiple time scales including seasonal to decadal time horizons.

7. A decision-making component that focuses on socio-economic impacts of human actions and is used to develop adaptation strategies. 


\section{DECADAL PREDICTIONS}

Over the last years, model projections, including for example those developed for the Intergovernmental Panel on Climate Change (IPCC) have privileged the hundred year timescales. These projections are determined primarily by external forcing mechanisms including the radiative forcing generated by the increasing atmospheric concentrations of greenhouse gases. For such long simulations, the initial conditions implemented in the models do not affect substantially the statistics of the future climate.

Much more difficult to perform are climate predictions on the seasonal to decadal time frame. In this case, one attempts to predict not only the long-term evolution of the climate variables, but also the year-to-year variability in the atmospheric and hydrological conditions. Here, the simulation depends directly on initial conditions, and specifically on the initial state of the ocean and ice system.

Decadal forecasting is still in its infancy. The predictability of the system over such timescales needs to be firmly established even if there are some encouraging signs in this direction. Decadal predictions, if successful, will prove invaluable for many sectors of society and for prevention of possible disasters, including heat waves, droughts, storms, hurricanes and flooding, damage to agriculture, forestry, fisheries, and water resources, spread of viruses and diseases, forest fires, etc. It will also be important for tourism, as well as for financial and insurance sectors.

Decadal climate prediction is a joint initial and boundary value problem. (initialization of climate state and climate forcing). Decadal to multi-decadal variability still not well understood. However, it is clear that the ocean will be at heart of such problem since some level of predictability is provided by the overturning circulation of the ocean. Full water column observations are therefore needed to initialize decadal prediction models. Long-term intensive observations in key regions of the ocean (interface between mid-latitude and tropical ocean, North Atlantic overturning circulation, Kurohio Oyashio Extension) are therefore needed.

Sustained time series observations will therefore be key for model initialization and model verification. Unfortunately, the ocean remains substantially undersampled. A suitable climate observing system should include a deep ocean component and cover the high latitudes. The maintenance of the current global system (Argo (Arrays for Real-time Geostrophic Oceanography), satellites) is a key requirement, but is not sufficient. Measurements of forcing fluxes at the airsea and land-sea boundaries are an important component of this observation system.
As the demand for biogeochemical data is increasing rapidly, in particular to initialize carbon cycle models, additional information should be provided. These include global and regional biomass (carbon) concentrations for the important plankton types, growth rates for all phytoplankton types as a function of temperature, light and nutrient concentrations, export of particulate organic carbon, decadal trends in surface ocean pCO2, decadal trends in sub-surface $\mathrm{O} 2$ concentration, etc..

To make full use of information provided by a global observing system, improved data assimilation systems need to be further developed. Specifically, the simultaneous observations and assimilation of quantities in coupled compartments of the Earth system should be regarded as an urgent necessity, even if it remains a difficult challenge.

\section{CONCLUSIONS}

The development of climate models will remain a focus in the coming decades with emphasis on reducing current and well-known biases, and on increasing spatial resolution for the representation of key atmospheric and ocean processes (cloud resolution, eddy resolution). At the same time, the interest is moving from century-long projections to seasonal-to-decadal climate predictions. Many challenges will need to be met before full decadal high-resolution prediction systems become operational and meet the growing demands of society.

No medium- or long-term forecast of the physical climate system and of the Earth system can be developed without incorporating a detailed ocean component in climate models. Observations are also essential to understand the relations between ocean dynamics, physics, biogeochemistry, ecosystems and living marine resources. Forecasting require initial conditions, whose quality will depend on the quality of observations and (coupled) data assimilation systems. The ocean remains under-sampled in spite of progress made in the last years. A well-designed integrated ocean observing system is essential for climate prediction on decadal timescales and will support societal needs. Climate Services will make use of such observational data.

\section{ACKNOWLEDGEMENTS.}

The Climate Service center is managed by the GKSS (Helmholtz-Zentrum Geesthacht Centre for Materials and Coastal Research) Research Center in Geestacht, Germany. The National Center for Atmospheric Research is operated by the University Corporation for Atmospheric Research and sponsored by the National Science Foundation in the USA. 